\title{
Antibiotic prescribing pattern in different clinical departments at Kathmandu Medical College Teaching Hospital
}

\author{
Dixit SM', Shrestha B²
}

'Sanjaya Mani Dixit, Lecturer; ${ }^{2}$ Binaya Shrestha, Assistant Professor; Department of Pharmacology, Kathmandu Medical College Teaching Hospital, Sinamangal, Kathmandu, Nepal

\begin{abstract}
Background: Antibiotics have transformed the practice of medicine for good; by making once lethal infections treatable. However, antimicrobials have been overused both in prophylaxis and therapy. Patients unnecessarily exposed to antibiotics are at risk for serious adverse events while increasing the chances of antimicrobial resistance. Patients with infections caused by drug-resistant bacteria are at increased risk of worse clinical outcomes and even death. It is therefore imperative to use our arsenal of antibiotics wisely.

Objectives: A prospective cross sectional study was carried out with the aim of identifying prescription pattern of antibiotics in different medical units at Kathmandu Medical College Teaching Hospital, Sinamangal. Investigating the prescription pattern of doctors and providing them necessary feedback plays a vital role in improving the prescription quality and promoting the rational prescription pattern.

Methodology: A study was carried out on in-patient prescription data collected at random from patients admitted in different clinical departments at the hospital. Information on medication use was obtained for the patients prescribed at least one antibiotic during their hospital stay. The antibiotic prescribing practices of different departments was compared by using the percentage method.

Results: The antibiotics most commonly prescribed were Ceftriaxone, Amoxicillin + Cloxacillin, Azithromycin, and Cefixime among others. Ceftriaxone was found to be prescribed in 108 cases accounting $16.8 \%$ of the total antibiotic prescribed.

Conclusion: The study highlights the current practice of antibiotics usage in clinical settings. Based on the current study it was concluded that Cephalosporins are the mostly used antibiotics in in-patient settings.
\end{abstract}

Key words: Antibiotics; Antimicrobial resistance; Antimicrobial stewardship; Drug utilization review; Prescription monitoring

DOI: http://dx.doi.org/10.3126/jkmc.v7i1.20624

\section{INTRODUCTION}

T

The advent of antibiotics has turned lethal infections

into treatable ones and has since then saved numerous lives. Antibiotics and vaccines together have drastically reduced mortality due to infectious diseases. Unfortunately, antibiotics, the miracle drugs of the $20^{\text {th }}$ century are used extensively and even unnecessarily.

The overuse of antibiotics both in prophylaxis and therapy is seen all over the world. The global antibiotic

Address for correspondence

Sanjaya Mani Dixit

Lecturer, Department of Pharmacology

Kathmandu Medical College Teaching Hospital,

Sinamangal, Kathmandu, Nepal

E-mail: sanjayadixit@gmail.com consumption has soared by $36 \%$ in the 2000 to 2010 period'. Center for Disease Control (CDC) in United States claims that a large percentage of antibiotics are misused in outpatient settings and that every 1 in 3 antibiotic prescriptions are unnecessary². A 2006 to 2012 study in inpatients conducted at over 300 hospitals in United States showed that over 55\% of patients received antibiotics during their stay ${ }^{3}$. Extensive mistreatment with antimicrobial drugs has been reported in the recent years, and nearly half of all antibiotic drug prescriptions have been found to be poorly selected ${ }^{4}$. Thus extensive use of antibiotics has resulted in unnecessary exposure to antibiotics, placing patients at risk for serious adverse effects while increasing antimicrobial resistance (AMR). There is a causal association between antimicrobial usage in hospital and antimicrobial resistance ${ }^{5}$. The growth of 
multiple-drug-resistant (MDR) bacterial pathogens can be traced to the overuse of broad-spectrum antimicrobial products both prophylactically and therapeutically ${ }^{6}$. Patients with infections caused by drug-resistant bacteria are at increased risk of worse clinical outcomes and even death, and consume more health-care resources than patients infected with non-resistant strains of the same bacteria ${ }^{7}$. Antibiotic resistance is not only a problem for the individual patient, it also reduces the effectiveness of established treatment and has become a major threat to public heath by increasing the complexity and cost of treatment and reducing the probability of successful outcome $^{8}$. With the antimicrobial resistance emerging as a global problem, and with more and more drug resistant strains of bacteria on the rise, it is getting imperative that we use our arsenal of antibiotics wisely to prevent the microbes from turning into drug resistant superbugs. In February 2017, WHO published a list of world's most dangerous superbugs for which new antibiotics are urgently required ${ }^{9}$. Interventions to promote rational antibiotic use are therefore crucial for preserving the effectiveness of available drugs ${ }^{10}$.

Developing and following antibiotic prescribing guidelines and protocols is a well established intervention to maintain effectiveness of current drugs $^{11}$. Another intervention aimed at decreasing the overuse of antibiotics and optimizing their rational and prudent use is the commencement of antibiotic stewardship programs (ASPs) in hospitals ${ }^{12}$. But majority of the hospitals here in Nepal lack proper guidelines and protocols on antibiotic use. Also the concept of antibiotic stewardship program is relatively new here ${ }^{13}$. It is thus solely the decision of the clinician to decide on the antibiotic therapy. Thereby, in absence of ASPs and antibiotic prescribing guidelines, the antibiotics can be overused and misused even in instances where they are not absolutely needed.

Conducting drug utilization research can provide useful information to health care providers and policy makers ${ }^{14}$. The study of the antibiotic prescribing patterns helps to monitor, evaluate and suggest changes in the clinician's prescription habits for efficient patient care ${ }^{15}$. This study was carried out to describe the types and characteristics of antibiotics prescribed in different clinical wards and to identify prescribing pattern of antibiotics in $\mathrm{KMCTH}$, a tertiary care teaching hospital in Kathmandu, Nepal.

\section{METHODOLOGY}

A cross-sectional study was carried out on in-patient prescription data. The data was collected at random from patient medical case records (patient cardex) for patients admitted in major clinical departments namely Medicine, Gynecology, Surgery, Paediatrics, Orthopaedics, ENT and Neurosurgery at Kathmandu Medical College Teaching Hospital (KMCTH) in Sinamangal from December 2016 to July 2017. Inpatients (both sexes) admitted in different clinical departments at KMCTH were included in the study. Patients brought to the emergency department, who died during their treatment who left against medical advice and patients in Intensive Care Unit (ICU), Critical Care Unit (CCU) were excluded from the study. Information on medication use was obtained from 388 inpatients prescribed at least one antibiotic during their stay in the hospital. The following information was noted for each of the patients including patient age, sex, diagnosis, specialty, and the prescribed antibiotic related information such as name of antibiotics, dose and their dosage forms. The data was then entered into and analyzed through Microsoft Excel.

An approval from the Institutional Review Committee of KMCTH was obtained prior to the study. The antibiotic prescribing practices of different departments was compared by using the percentage method. Analysis was carried out further to identify the number of cases where antibiotics were prescribed as monotherapy and the cases where multiple antibiotics were prescribed. The antibiotic used in maximum number of cases was analyzed and also department wise survey of the top five antibiotics used by the department was done.

\section{RESULTS}

A total of 37 different antibiotics were prescribed. The most common antibiotics prescribed in our study were Ceftriaxone, Amoxicillin+Cloxacillin, Azithromycin, Cefixime, Cloxacillin among others. Ceftriaxone was found to be prescribed in 108 cases accounting for $16.8 \%$ of total antibiotics prescribed as shown in Table 1. Ceftriaxone was among the top three most prescribed antibiotics in all the five clinical departments prescribing maximal antibiotics.

\section{DEPARTMENT WISE SURVEY OF ANTIBIOTICS USED}

The Department of Gynaecology and Obstetrics, Medicine, Surgery, Paediatrics and Orthopaedics respectively used maximal antibiotics. The top three antibiotics prescribed by the different departments are shown in Figure 1.

The Department of Gynaecology and Obstetrics used the combination of Amoxicillin and Cloxacillin the most. Ceftriaxone was the top most prescribed antibiotic in the Department of Medicine and the Department 
of Surgery. Similarly, the maximal used antibiotics in the Department of Paediatrics and the Department of Orthopaedics were Amoxicillin+ Clavulanic acid and Cefuroxime respectively.

\section{MONOTHERAPY VERSUS POLYTHERAPY}

Among 388 prescriptions for antibiotics 199 (51.3\%) were prescribed as monotherapy and 189 (49.7\%) were prescribed as polytherapy. Further subdividing the polytherapy groups it was seen that 142 (36.6\%) prescriptions contained two antibiotics, 34 (8.8\%) prescriptions contained three antibiotics and 13 (3.4\%) prescriptions containedfour or more than four antibiotics as shown in Figure 2. These accounted for a sum total of 641 antibiotics prescribed for 388 inpatients.

\section{AGE AND SEX WISE USAGE OF ANTIBIOTICS}

Out of the total 388 prescriptions for antibiotics it was seen that 164 belonged male patients and 224 belonged to female patients. We also found that the antibiotics were prescribed maximally for the age group 21-30 years old patients as shown in Figure 3.

\section{ROUTE OF ADMINISTRATION}

Among the 641 antibiotics prescribed 338 were administered in injection form and 295 prescriptions were given orally in either tablet, capsule, suspension or syrup form while remaining eightantibiotics were administered topically. Figure 4 shows the different routes through which the antibiotics were administered to the patients.

\section{Table 1: Top ten antibiotics prescribed for the inpatients}

\begin{tabular}{|c|c|c|c|}
\hline S. No. & $\begin{array}{l}\text { Commonly prescribed } \\
\text { Antibiotics }\end{array}$ & Frequency & Percentage \\
\hline 1 & Ceftriaxone & 108 & $16.8 \%$ \\
\hline 2 & Amoxicillin + Cloxacillin & 83 & $12.9 \%$ \\
\hline 3 & Azithromycin & 64 & $10.0 \%$ \\
\hline 4 & Cefixime & 41 & $6.4 \%$ \\
\hline 5 & Cloxacillin & 39 & $6.1 \%$ \\
\hline 6 & Ciprofloxacin & 34 & $5.3 \%$ \\
\hline 7 & Cefpodoxime & 29 & $4.5 \%$ \\
\hline 8 & $\begin{array}{l}\text { Amoxicillin }+ \\
\text { Clavulanic acid }\end{array}$ & 28 & $4.4 \%$ \\
\hline 9 & Amikacin & 25 & $3.9 \%$ \\
\hline \multirow[t]{3}{*}{10} & Cefotaxime & 23 & $3.6 \%$ \\
\hline & Others (27 antibiotics) & 167 & $26.1 \%$ \\
\hline & Total & 641 & $100.0 \%$ \\
\hline
\end{tabular}

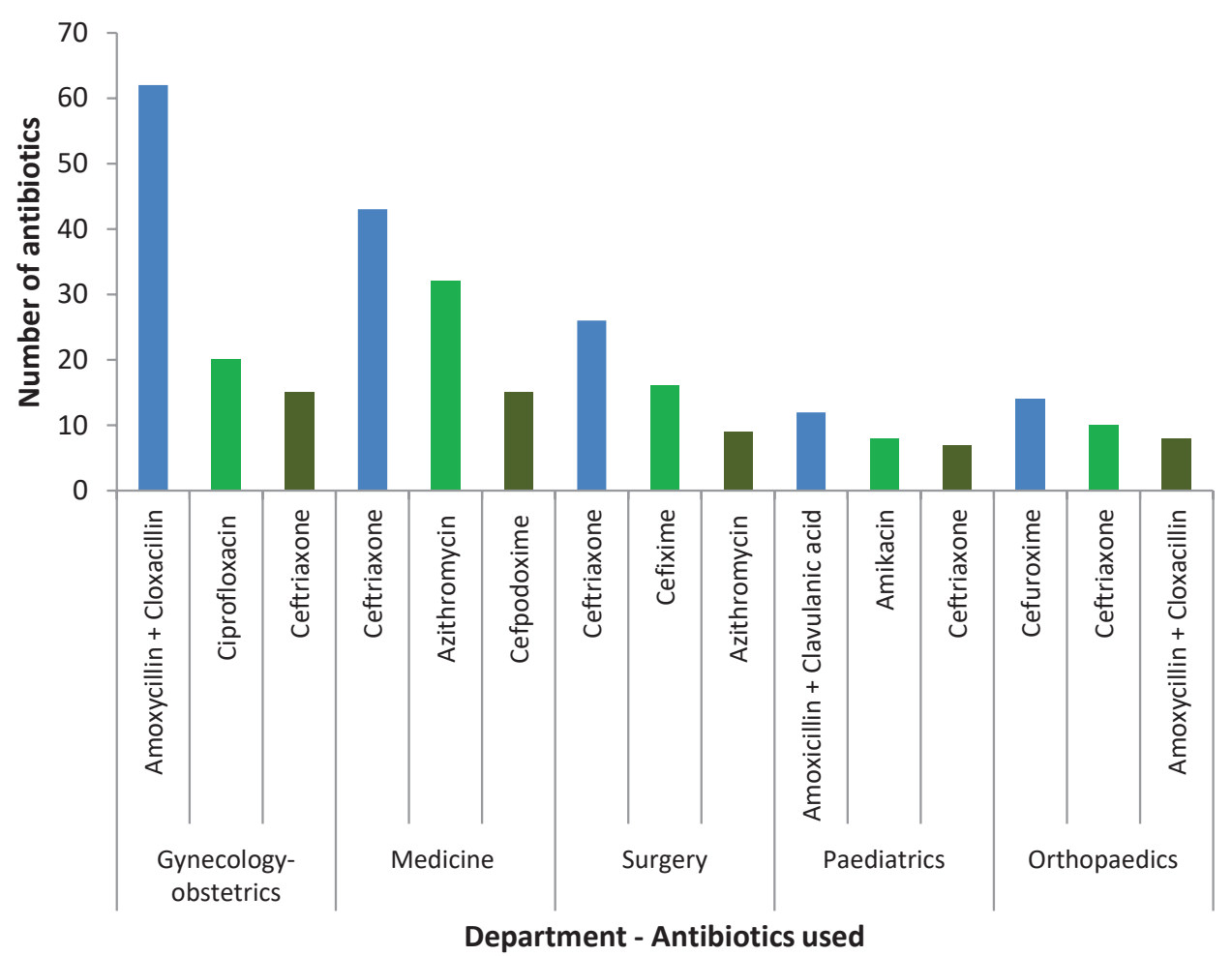

Figure 1: Top 3 antibiotics prescribed by top 5 antibiotic prescribing departments 


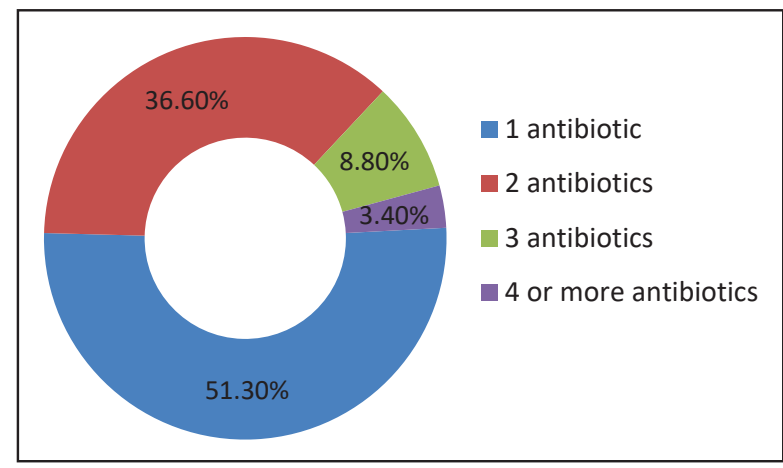

Figure 2: Monotherapy Vs Polytherapy

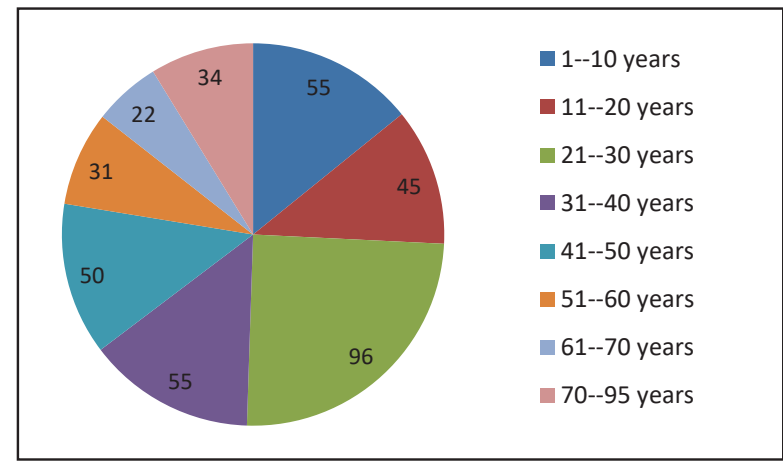

Figure 3: Antibiotic use according to age groups

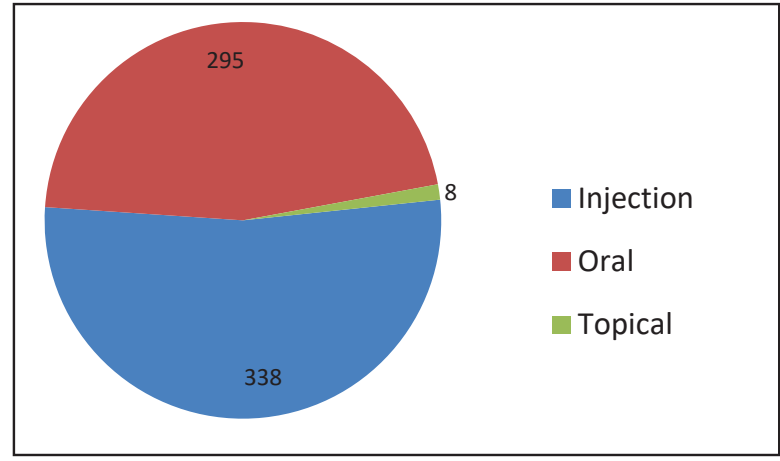

Figure 4: Route of administration of antibiotics

\section{DISCUSSION}

The most commonly prescribed antibiotics comprised of Ceftriaxone, Amoxicillin + Cloxacillin, Azithromycin, Cefixime and Cloxacillin. Ceftriaxone, the third generation Cephalosporin, was used in 108 cases accounting for $16.8 \%$ of all antibiotics used. A similar study conducted in 2013 in ICU patients in India also showed that Ceftriaxone was prescribed in most of the patients and accounted for $23 \%$ of antimicrobial therapy ${ }^{16}$. Another study conducted by Vandana $A B$ et al. in 2010 observed that Cefotaxime was the most prescribed antibiotic ${ }^{17}$. Other studies have also similarly pointed out that Cephalosporins are the most widely utilized first line antibiotics ${ }^{18}$. In 2002 a study conducted by Shankar et al. in Nepal showed Ampicillin, Amoxicillin, Metronidazole, Ciprofloxacin and Benzylpenicillin as the five most commonly prescribed antibiotics ${ }^{15}$. Similarity in the type of antibiotic prescribed in the different studies points out that India and Nepal have similar disease prevalence and similar prescribing patterns. This reflects a change in prescription pattern over a period of time which is due to change in the pattern of infections with resistant organisms and the varying susceptibility of the microorganisms to antibiotics.

The fixed dose combination (FDC) of Amoxicillin+Cloxacillin was the second most used antibiotic in our study. When compared to the study by Shankar et al. conducted in 2002, Amoxicillin alone was ranked as the second most prescribed antibiotic ${ }^{15}$. The reason for the increased use of Amoxicillin combination with the antistaphylococcal drug Cloxacillin is attributed to increased infections by Beta-lactamase producing strains of Staphlylococci as a result of increased antimicrobial resistance.

In our study it was observed that the patients admitted in Gynaecology-Obstetrics were prescribed maximum antibiotics. This may be due to the fact that 29 percent of patients were from Gynaecology-Obstetrics Department. This was followed by Medicine and Surgery departments which comprised 21 percent and 16 percent of the patients respectively. Also another finding seen in our studywas that the age group between 21-30 years was the group for which antibiotics were mostly prescribed, this can be related to the fact that most of the pregnant women belonged to this age group and while undergoing caesarean section were given some antibiotics prophylactically.

Our study showed that Amoxicillin+ Cloxacillin, Ciprofloxacin and Ceftriaxone were the most prescribed antibiotics by the department of Gynecology and Obstetrics. Penicillins and Cephalosporins high use may be attributed to their relative safety in obstetric use. Ceftriaxone may have been used more for the treatment of Sexually Transmitted Diseases (STDs) as per Center for Disease Control and Prevention (CDC) recommendations ${ }^{19}$. A 5 year long study done in Thailand found Ampicillin to be equally effective as Ceftriaxone in preventing caesarean section surgical site skin infections ${ }^{20}$. There seems to be a need to carry on similar studies here in the future so that narrow spectrum drugs can replace the broad spectrum ones in clinical use, thereby decreasing the emergence of AMR. 
Both Medicine and Surgery departments used Ceftriaxone the most. Basnyat et al. in a 2015 study mentioned that several studies across different healthcare facilities showed evidence of inappropriate prescribing of antibiotics in Nepal and it was particularly true for Ceftriaxone amongst others ${ }^{21}$. Ceftriaxone is overused not only for fever of unknown origin, but also for meningitis, pneumonia, urinary tract infections, and many other infections. The overuse of Ceftriaxone has caused common gram-negative bacteria, like Klebsiella and Escherichia coli to acquire antimicrobial resistance through production of 'Extended-Spectrum BetaLactamases' (ESBL) rendering the organisms no longer susceptible to Ceftriaxone ${ }^{22}$.

Azithromycin a broad spectrum macrolide was used more by Medicine and Surgery. This may be due to the fact that Azithromycin is preferred in respiratory, enteric and genitourinary infections the commonly occurring infections in Nepal ${ }^{23,24}$. CDC also recommends Azithromycin for the treatment of different STDs ${ }^{19}$. Azithromycin being a drug with long plasma half life achieving higher tissue concentrations and having broad spectrum of action may be preferred in various surgeries ${ }^{25,26}$.

We found that the FDC of Amoxicillin+Clavulanic acid was the top most used antibiotic by the department of Paediatrics, followed by Amikacin and Ceftriaxone. This seems to be in line with CDC which has ranked this FDC as the first line antibiotic in different paediatric infections like- sinusitis, acute otitis media and urinary tract infections ${ }^{27}$.

Cefuroxime, Ceftriaxone and Amoxicillin+Cloxacillin combination were the most used medicines by Orthopaedics department. Literature review also shows that intravenous Cefuroxime is the most commonly used antibiotic for both pre and post-operative cases for patients undergoing internal fixation of fractures ${ }^{28,29}$. Yeap et al. suggested that Cephalosporins are by far the most popular choice of antibiotics for prophylaxis in orthopaedic surgeries ${ }^{28}$, which was also the case in this study.

In our study, we found that 199 prescriptions out of 388 were prescribed as monotherapy for antibiotics. Prescribing antibiotics as monotherapy is a good practice aimed at decreasing the antimicrobial resistance. Since the modern day antibiotics can cover a spectrum of organisms in mixed infections, single agents are preferred today. However, combinations of antibiotics in different scenarios are more effective in preventing the emergence of resistance and at times also show synergistic effects ${ }^{30}$. At times when empiric coverage is a must, combination therapy may be necessary ${ }^{31}$. The use of combination therapy involving more than 3 agents was seen in only 47 prescriptions, this shows that antimicrobial therapy use was fairly rational.

Out of 641 antibiotics prescribed 338 were administered in injection form and 295 prescriptions were given orally while remaining eight antibiotics were administered topically. The relatively high use of injectable form of antibiotics can be because our study was conducted in in-patients where the use of injectable medicines is relatively high owing to the severity of the cases. This may also be attributed to the belief in the superiority of intravenous antibiotics which is widespread among health professionals and patients alike ${ }^{32}$. It could also be because the physicians usually opt for IV medications at first and continue them till patient discharge as suggested by Cyriac et al..$^{33}$ This is also so in-part because our study identified Ceftriaxone as the mostly used antibiotic which comes in injectable form only. The high use of injectable drugs can sometimes contribute to infections and even lead to sepsis, especially if they are given via intramuscular route ${ }^{34}$. Though not adequately investigated the chances of injectable drugs causing healthcare associated infections is high in our cases because they are not prepared in aseptic laminar flow hoods, rather at the patient bed-side itself, where the load of pathogenic organisms is high.

In South East Asia region the antibiotic prescribing is relatively high owing to higher incidence of infectious diseases owing to environmental factors and lack of proper sanitation measures. We also have a high burden of water borne diseases. We also cannot deny the fact that even the patients by the time they require hospitalization are exposed to different antibiotics as a result of self medication from the chemists shop before seeing a medical practitioner itself ${ }^{35}$. To discourage this sort of practices WHO is celebrating World Antibiotic Awareness Week 2017 with the slogan: Our time with antibiotics is running out. Always seek the advice of a healthcare professional before taking antibiotics ${ }^{36}$. With Nepal lacking registered pharmacists in most of the drug stores, patient counseling with regards to proper use of antibiotics is often missing and patients many a times do not take the complete course of antibiotics as prescribed. This coupled with the ease of obtaining antibiotics from drug stores in Nepal is an important contributor to $\mathrm{AMR}^{35}$. 
As a strategy to decrease the incidence of antimicrobial resistance a certain antibiotics should be reserved as "last hope" antibiotics which only senior consultants should be allowed to prescribe and antimicrobial stewards should have their final say on use of such drugs. UK had similarly listed Colistin as the last hope antibiotic, whose use is now soaring with the rise of the infections caused by the superbugs ${ }^{37}$. Global Antibiotic Resistance Partnership (GARP)-Nepal in outlining the prime strategies to improve antibiotics use at national level has focused on the importance of improving hospital infection control and establishing antimicrobial stewardship ${ }^{21}$.Antimicrobial stewardship program is known to promote the appropriate use of antimicrobials, improve patient outcomes, reduce microbial resistance, and decrease the spread of infections caused by multidrug-resistant organisms ${ }^{12}$. Medical colleges are best suited to work as exemplary models to establish and run infection prevention and control programs and antimicrobial stewardship programs with adequate professional manpower to accomplish the task.

\section{CONCLUSION}

Based on the results it was concluded that the Cephalosporin group of antibiotics is the most frequently prescribed antibiotics, with Ceftriaxone being the

\section{REFERENCES}

1. Franko E. Global antibiotic consumption soars $36 \%$ in 10 years, raising concerns about superbugs - Center for Disease Dynamics, Economics \&amp; Policy (CDDEP) [Internet]. CDDEP. 2014 [cited 2018 Jan 31]. Available from: https://www.cddep.org/blog/posts/ global_antibiotic_consumption_soars_36_10_ years_raising_concerns_about_superbugs/

2. CDC: 1 in 3 antibiotic prescriptions unnecessary | CDC Online Newsroom | CDC [Internet]. Centers for Disease Control and Prevention. 2016 [cited 2017 Nov 30]. Available from: https://www.cdc. gov/media/releases/2016/p0503-unnecessaryprescriptions.html

3. Baggs J, Fridkin SK, Pollack LA, Srinivasan A, Jernigan JA. Estimating National Trends in Inpatient Antibiotic Use Among US Hospitals From 2006 to 2012. JAMA Intern Med [Internet]. 2016 Nov 1 [cited 2018 Mar 31];176(11):1639-48. Available from: http://archinte. jamanetwork.com/article.aspx?doi=10.1001/ jamainternmed.2016.5651

4. Al Shimemeri A, Al Ghadeer H, Memish Z. Antibiotic utilization pattern in a general medical ward of a tertiary medical center in Saudi Arabia. Avicenna J Med [Internet]. 2011 Jul [cited 2017 Jun 30];1(1):8- maximally used drug. Infectious disease burden being high here, it was seen that the antibiotics is highly used in hospital settings. Lack of development of prescribing guidelines and protocols for prescribing the antibiotics in individual hospitals is an important factor contributing to the misuse and over use of antibiotics thereby leading to antimicrobial resistance. There appears to be a crucial need for starting antibiotic stewardship program which will monitor the patient's on antibiotic therapy in real time and give constructive feedback to the physicians regarding their choice of antibiotics in therapy. Then only we can attain the mission of ensuring that every patient gets optimal antibiotic therapy. Hospitals' lacking antibiotic prescribing guidelines and protocols and the delay in initiating the antibiotic stewardship program is a huge problem in our hospital settings and we should soon be able to overcome this milestone for threat of antimicrobial resistance is real.

\section{ACKNOWLEDGEMENTS}

We would like to extend our sincere gratitude to Professor Dr. Hemang Dixit for going through the article multiple times and providing us constructive feedback by to help us develop the article in its final form. Finally, a note of thanks to all who extended their helping hands in different stages of data collection.

11. Available from: http://www.ncbi.nlm.nih.gov/ pubmed/23210003

5. Randad RD, Bhagwate ST, Inamdar MK. Drug utilization study of some antibiotic in indoor setting at tertiary care teaching hospital in Central India : a descriptive study. IJBCP [Internet]. 2017;6(5):1123-6. Available from: http://www.ijbcp.com/index.php/ ijbcp/article/view/1457

6. GootzTD. The global problem of antibiotic resistance. Crit Rev Immunol [Internet]. 2010 [cited 2017 Jun 30];30(1):79-93. Available from: http://www.ncbi. nlm.nih.gov/pubmed/20370622

7. WHO Antimicrobial resistance [Internet]. WHO. World Health Organization; 2016 [cited 2017 Jun 30]. Available from: http://www.who.int/mediacentre/ factsheets/fs194/en/

8. Holloway K, Dijk L van. The world medicines situation: rational use of medicines. World Med Situat. $2011 ; 2(2): 24$.

9. $\mathrm{WHO} \mid \mathrm{WHO}$ publishes list of bacteria for which new antibiotics are urgently needed [Internet]. WHO. World Health Organization; 2017 [cited 2017 Nov 13]. Available from: http://www.who.int/mediacentre/ news/releases/2017/bacteria-antibiotics-needed/ en/ 
10. Rogawski ET, Platts-Mills JA, Seidman JC, John $S$, Mahfuz $M$, Ulak $M$, et al. Use of antibiotics in children younger than two years in eight countries: a prospective cohort study. Bull World Health Organ [Internet]. 2017;95(1):49-61. Available from: http://www.who.int/entity/bulletin/ volumes/95/1/16-176123.pdf

11. Center for Disease Dynamics Economics \& Policy. The State of the world's antibiotics 2015. Cent Dis Dyn Econ Policy, CDDEP Washington, DC. 2015;1-84.

12. Doron S, Davidson LE. Antimicrobial stewardship. Mayo Clin Proc [Internet]. 2011 Nov [cited 2018 Mar 31];86(11):1113-23. Available from: http://www. ncbi.nlm.nih.gov/pubmed/22033257

13. Veve MP, Kaljee LM, Prentiss T, Joshi RD, Rai SM, Shrestha B, et al. Implementing Antimicrobial Stewardship in Two Community Nepali Hospitals. Open Forum Infect Dis [Internet]. 2017 [cited 2018 Mar 30];4(suppl_1):S20-S20. Available from: http://academic.oup.com/ofid/article/4/suppl_1/ S20/4293923/Implementing-AntimicrobialStewardship-in-Two

14. Dp S, Phunyalb, Maharjanm, Mandals, Mahatosc, Gp R. Utilization pattern of antimicrobial agents and its culture sensitivity pattern in intensive care units in a tertiary care center in eastern Nepal. J Nepal Med Coll [Internet]. 2015 [cited 2017 Jun 30];17(3-4):107-12. Available from: http://nmcth.edu/images/gallery/ Original Article/DP_Sarraf_et_al.pdf

15. Shankar R, Partha P, Shenoy N, Easow J, Brahmadathan K. Prescribing patterns of antibiotics and sensitivity patterns of common microorganisms in the Internal Medicine ward of a teaching hospital in Western Nepal: a prospective study. Ann Clin Microbiol Antimicrob [Internet]. 2003 Jul 16 [cited 2017 Nov 30];2(1):7. Available from: http://www. ncbi.nlm.nih.gov/pubmed/12904265

16. Pandiamunian J, Somasundaram G. A study on prescribing pattern of anti microbial agents in the medical intensive care unit of a tertiary care teaching hospital in Puducherry union territory, South India. Int J Pharm Pharm Sci [Internet]. 2014;6(3):235. Int J Pharm Pharm Sci [Internet]. 2014;6(3):235-8. Available from: http://www.ijppsjournal.com/ Vol6lssue3/9047.pdf

17. Badar VA, Navale SB. Study of prescribing pattern of antimicrobial agents in medicine intensive care unit of a teaching hospital in Central India. J Assoc Physicians India [Internet]. 2012 Apr [cited 2017 Nov 30];60:20-3. Available from: http://www.ncbi.nlm. nih.gov/pubmed/23029737

18. Fair RJ, Tor Y. Antibiotics and bacterial resistance in the 21st century. Perspect Medicin Chem [Internet].
2014 [cited 2017 May 26];6:25-64. Available from: http://www.ncbi.nlm.nih.gov/pubmed/25232278

19. CDC Treatment Guidelines [Internet]. Atlanta; 2015 [cited 2018 Mar 18]. Available from: https://www. cdc.gov/std/tg2015/2015-pocket-guide.pdf

20. Assawapalanggool S, Kasatpibal N, Sirichotiyakul S, Arora R, Suntornlimsiri W, Apisarnthanarak A. The efficacy of ampicillin compared with ceftriaxone on preventing cesarean surgical site infections: an observational prospective cohort study. Antimicrob Resist Infect Control [Internet]. 2018 [cited 2018 Mar 18];7:13. Available from: http://www.ncbi.nlm.nih. gov/pubmed/29387347

21. Basnyat B, Pokharel P, Dixit S, Giri S. Antibiotic Use, Its Resistance in Nepal and Recommendations for Action: A Situation Analysis. J Nepal Health Res Counc [Internet]. 2015;13(30):102-11. Available from: http://ovidsp.ovid.com/ovidweb.cgi?T=JS\&PA $\mathrm{GE}=$ reference $\& D=$ prem \&NEWS $=\mathrm{N} \& A N=26744193$

22. Basnyat B. Dodging the silver bullet. Nepalitimes [Internet]. 2012 Nov [cited 2018 Mar 15]; Available from: http://archive.nepalitimes.com/news. php?id=19787\#.Wqpb_8NublU

23. McMullan BJ, Mostaghim M. Prescribing azithromycin. Aust Prescr [Internet]. 2015 Jun [cited 2018 Mar 15];38(3):87-9. Available from: http://www. ncbi.nlm.nih.gov/pubmed/26648627

24. Karki R. Management of disease outbreak in Nepal. Lancet (London, England) [Internet]. 2015 Jul 25 [cited 2018 Mar 18];386(9991):335-6. Available from: http://www.ncbi.nlm.nih.gov/pubmed/26227460

25. Lamont RF, Sobel JD, Kusanovic JP, Vaisbuch E, Mazaki-Tovi S, Kim SK, et al. Current debate on the use of antibiotic prophylaxis for caesarean section. BJOG [Internet]. 2011 Jan [cited 2018 Mar 18];118(2):193201. Available from: http://www.ncbi.nlm.nih.gov/ pubmed/21159119

26. Nakayama I. Clinical studies of azithromycin, a new macrolide antibiotic, for infections in the field of surgery. Jpn J Antibiot [Internet]. 2000 Jun [cited 2018 Mar 19];53 Suppl B:82-90. Available from: http://www.ncbi.nlm.nih.gov/pubmed/12572091

27. Pediatric Treatment Recommendations / Community | Antibiotic Use | CDC [Internet]. CDC . 2017 [cited 2018 Mar 18]. Available from: https://www.cdc.gov/ antibiotic-use/community/for-hcp/outpatient-hcp/ pediatric-treatment-rec.html

28. Yeap JS, Lim JW, Vergis M, Au Yeung PS, Chiu CK, Singh $\mathrm{H}$. Prophylactic antibiotics in orthopaedic surgery: guidelines and practice. Med J Malaysia [Internet]. 2006 Jun [cited 2018 Mar 18];61(2):1818. Available from: http://www.ncbi.nlm.nih.gov/ pubmed/16898309 
29. Dhammi IK, UI Haq R, Kumar S. Prophylactic antibiotics in orthopedic surgery: Controversial issues in its use. Indian J Orthop [Internet]. 2015 [cited 2018 Mar 18];49(4):373-6. Available from: http://www.ncbi.nlm.nih.gov/pubmed/26229155

30. Barriere SL. Monotherapy versus Combination Antimicrobial Therapy: A Review. Pharmacother J Hum Pharmacol Drug Ther [Internet]. 1991 Mar 4 [cited 2018 Mar 16];11(2P2). Available from: http://onlinelibrary.wiley.com/ doi/10.1002/j.1875-9114.1991.tb02621.x/abstract

31. Patel SM, Saravolatz LD. Monotherapy Versus Combination Therapy. Med Clin North Am [Internet]. 2006 Nov [cited 2018 Mar 16];90(6):1183-95. Available from: http://www.ncbi.nlm.nih.gov/ pubmed/17116443

32. Li HK, Agweyu A, English M, Bejon P. An unsupported preference for intravenous antibiotics. PLoS Med [Internet]. 2015 May [cited 2018 Mar 14];12(5):e1001825. Available from: http://www. ncbi.nlm.nih.gov/pubmed/25992781
33. Cyriac JM, James E. Switch over from intravenous to oral therapy: A concise overview. J Pharmacol Pharmacother [Internet]. 2014 Apr [cited 2018 Mar 15];5(2):83-7. Available from: http://www.ncbi.nlm.nih. gov/pubmed/24799810

34. Risks of intramuscular injection | Dopinglinkki [Internet]. [cited 2017 Dec 13]. Available from: https:// dopinglinkki.fi/en/info-bank/doping-substances/risksintramuscular-injection

35. Wachter DA, Joshi MP, Rimal B. Antibiotic dispensing by drug retailers in Kathmandu, Nepal. Trop Med Int Heal. 1999;4(11):782-8.

36. WHO | Posters: World Antibiotic Awareness Week 2017 [Internet]. WHO. World Health Organization; 2017 [cited 2017 Nov 13]. Available from: http://www.who.int/ campaigns/world-antibiotic-awareness-week/2017/ posters/en/

37. Use of "last hope" antibiotic soaring in English hospitals - The Bureau of Investigative Journalism [Internet]. The Bureau of Investigative Journalism. 2017 [cited 2017 Dec 13]. Available from: https:// www.thebureauinvestigates.com/stories/2017-02-24/ colistin-antibiotic-resistance-superbugs-hospitals 